Nachhaltigkeit im deutschen Forschungssystem

\title{
Noch ein langer Weg zur nachhaltigen Wissenschaft
}

\author{
Wie innovativ ist das deutsche Forschungs- \\ system im Hinblick auf die Herausforderung \\ „Nachhaltige Entwicklung“ eigentlich selbst? \\ Eine gerade erschienene Studie zur Nach- \\ haltigkeit im deutschen Wissenschafts- und \\ Hochschulsystem zeigt Stand und \\ Herausforderungen auf. \\ Von Uwe Schneidewind
}

\begin{abstract}
ustainability Science is an emerging discipline that seeks to -understand the interactions within and between global, social and human systems, the complex mechanisms that lead to the degradation of these systems, and the concomitant risks to human well-being and security. It also seeks to provide the vision and methodology that will lead to the restoration of these systems," so kennzeichnen Steinfeld und Mino das Feld der Nachhaltigkeitswissenschaft und fügen im Anschluss hinzu: „A particular challenge is how to transform the educational system (...) to make this possible." (Steinfeld/Mino 2009)

Wo stehen wir in Deutschland im Hinblick auf eine so verstandene Nachhaltigkeitswissenschaft? Wo findet sich eine auf transdisziplinäre, das heißt eine auf System-, Ziel- und Transformationswissen zielende Forschung in den Hochschulen und außeruniversitären Forschungseinrichtungen, die den Herausforderungen einer nachhaltigen Entwicklung gerecht wird?
\end{abstract}

\section{Ernüchterndes Bild der Exzellenzinitiative}

Für den Stand einer nachhaltigen Wissenschaft im deutschen Forschungssystem sind die in den Jahren 2006 und 2007 durchgeführten Runden der Exzellenzinitiative ein interessanter Gradmesser. Alle deutschen Universitäten waren in enger Kooperation mit den großen außeruniversitären Forschungseinrichtungen aufgefordert, Anträge für Exzellenzcluster, Graduiertenschulen sowie universitätsumfassende Zukunftskonzepte zu stellen. Insgesamt werden bis zum Jahr 2013 für die am Ende bewilligten 39 Graduiertenschulen, 37 Exzellenzcluster und neun Zukunftskonzepte für die neun sogenannten Eliteuniversitäten 1,9 Milliarden Euro ausgegeben.

Angesichts der großen und flächendeckenden Resonanz auf die Exzellenzinitiative - die meisten Universitäten hatten sich mit Anträgen mindestens in einer der Ausschreibungslinien beteiligt - lieferte die Exzellenzinitiative einen sehr guten Über- blick darüber, wo die deutsche Wissenschaft ihre leistungsfähigsten Potenziale aufgebaut hat. Im Hinblick auf eine nachhaltige Wissenschaft ist das Ergebnis ernüchternd. So wäre zu erwarten gewesen, dass in einem Land wie Deutschland, das global eine politische Führungsrolle in Strategien für eine nachhaltige und klimagerechte Entwicklung übernommen hat, sich dies auch in den dominanten Forschungsthemen und -strategien widerspiegelt. Angesichts der Tatsache, dass die Herausforderung des Global Change integrierte Konzepte für einen nachhaltigen Wandel benötigt, wäre auf entsprechend akzentuierte transdisziplinäre Entwürfe und Kompetenzzentren aus dem Wissenschaftsbereich zu hoffen gewesen.

Der Blick auf die geförderten Graduiertenschulen und Exzellenzcluster sowie die neun Eliteuniversitäten enttäuscht diese Hoffnungen. Letztlich finden sich nur vier geförderte Cluster, die dominant Umweltthemen adressieren: das Kieler Cluster „Ozean der Zukunft“, das Bremer Cluster „The Ocean in the Earth System“, das Hamburger-Klima-Cluster „Integrated Climate System Analysis and Prediction" und das Aachener Cluster „Maßgeschneiderte Kraftstoffe aus Biomasse“. Ein näherer Blick auf die Cluster zeigt, dass hier naturwissenschaftlich-technische Perspektiven dominieren, eine integrierte naturwissenschaftlich-sozialwissenschaftliche Perspektive im Sinne einer transdisziplinären Forschung sich nur in Ansätzen findet.

Die im Rahmen der Exzellenzinitiative geförderten relevanten sozial- und geisteswissenschaftlichen Verbünde wie das Konstanzer Cluster „Kulturelle Grundlagen von Integration“ oder das Frankfurter Cluster „Formation of Normative Orders“ werfen Schlüsselfragen auf, die auch für den Nachhaltigkeitsdiskurs von Bedeutung sind, ohne diese Verbindung aber explizit vorzunehmen.

\section{Fragen der Nachhaltigkeit spielen eine geringe Rolle}

Ein ähnliches Bild liefert die Auswahl der neun geförderten Eliteuniversitäten, das heißt der Universitäten, deren Zukunftskonzept im Rahmen der Exzellenzinitiative ausgezeichnet wurde und von denen jede auf diese Weise rund 100 Millionen Euro, verteilt auf fünf Jahre, zum Ausbau ihrer Gesamtstrategie erhält. Diese neun Universitäten sind die Technische Universität München und die Ludwig-Maximilians-Universität München in Bayern, die Universitäten Freiburg, Heidelberg, Karlsruhe und Konstanz in Baden-Württemberg sowie die Rheinisch-Westfälische Technische Hochschule Aachen in Nord- 
rhein-Westfalen, die Universität Göttingen in Niedersachsen und die Freie Universität Berlin.

Die nähere Betrachtung dieser Universitäten zeigt, dass in ihnen die Potenziale für eine transdisziplinäre Nachhaltigkeitsforschung durchaus vorhanden sind. Wir finden Hochschulen mit einer differenziert ausgebildeten Umweltforschung (TU München, Universität Göttingen, Universität Freiburg), starken Ausprägungen von Gesellschaftsorientierung (FU Berlin) sowie einem beeindruckenden Maß an Interdisziplinarität (Universität Konstanz). Jedoch sind diese einzelnen Ingredienzien, derer es für eine Nachhaltigkeitsforschung bedarf, innerhalb sowie über die Exzellenzuniversitäten hinweg verstreut. Bei keinem der Gewinner der neun Zukunftskonzepte kommen sie zu einem herausragenden Profil einer "Sustainable University“ zusammen. In den Zukunftskonzepten spielen daher Fragen der Nachhaltigkeit bei allen neun Universitäten faktisch auch keine Rolle.

\section{Transdisziplinäre Verbünde sind die Ausnahme}

Letztlich sind diese Ergebnisse der Exzellenzinitiative nicht überraschend, da sie nur die Spitze der ansonsten geförderten Forschungspraxis abbilden. So gibt es bei den von der Deutschen Forschungsgemeinschaft (DFG) unterstützten koordinierten Forschungsverbünden (Sonderforschungsbereiche, kurz SFB, Transregios und Schwerpunktprogramme, kurz SPP) nur wenige Beispiele für transdisziplinäre Verbünde der Umweltund Nachhaltigkeitsforschung. Wie der Blick auf die Anfang 2009 aktuell gut 200 geförderten Sonderforschungsbereiche und knapp 50 Transregios zeigt, sind Verbünde einer transdiszplinären Nachhaltigkeitsforschung die Ausnahme. Eine davon ist beispielsweise der seit 2000 bestehende, an der Universität Hohenheim angesiedelte SFB 564 „Nachhaltige Landnutzung und ländliche Entwicklung in Bergregionen Südostasiens“.

Am offensten sind die Schwerpunktprogramme der DFG. Sie stellen die loseste Form der koordinierten Förderung unter einem gemeinsamen thematischen Dach dar. Zu dem übergeordneten Thema werden im Rahmen der sechsjährigen Laufzeit Ausschreibungsrunden zu Einzelanträgen initiiert. Unter den knapp 100 Schwerpunktprogrammen der DFG Anfang 2009 befanden sich durchaus einige mit explizitem Klimaund Nachhaltigkeitsbezug wie zum Beispiel das von Kiel koordinierte SPP 1162 „The impact of climate variability on aquatic ecosystems (AQUASHIFT)“, das von Kassel koordinierte SPP 1182 „Nachhaltiges Bauen mit Ultra-Hochfestem Beton (UHPC)“ oder das von Köln koordinierte SPP 1233 „Megastädte:
Informelle Dynamik des Globalen Wandels“ - jeweils mit einem sehr unterschiedlichen Grad an Inter- und Transdisziplinarität.

\section{Nachholbedarf auch im sonstigen Forschungssystem}

Auch bei den nationalen Wissenschaftsgemeinschaften, also den Instituten der Helmholtz-Gemeinschaft, der Leibniz-Gemeinschaft, der Fraunhofer-Gesellschaft und der Max-PlanckGesellschaft, zeigt sich ein ähnliches Bild. Insbesondere in der Helmholtz- und Leibniz-Gemeinschaft existieren zahlreiche Institute mit starkem, durchaus auch interdisziplinärem Umweltund Nachhaltigkeitsbezug wie zum Beispiel das Umweltforschungszentrum (UFZ) in Leipzig (Helmholtz) oder insbesondere das Potsdam Institut für Klimafolgenforschung (PIK, Leibniz). Die Helmholtz-Gemeinschaft legt zudem seit einigen Jahren institutsübergreifende Forschungsschwerpunkte zu umwelt- und nachhaltigkeitsrelevanten Themen auf. In industrierelevanten Bereichen wie insbesondere der Energieforschung gilt das auch für die Fraunhofer-Gemeinschaft. Dennoch bleiben die meisten der Programme naturwissenschaftlich und technologisch orientiert und sind von den Anforderungen an eine "Sustainability Science“ noch weit entfernt. Für die MaxPlanck-Gesellschaft gilt dies aufgrund ihres Grundlagenforschungsauftrages ohnehin.

Schließlich ist zu betonen, dass die gerade skizzierten Bereiche der Forschung an den Hochschulen sowie der national geförderten außeruniversitären Forschungsinstitute nicht einmal ein Drittel des Gesamtvolumens des deutschen Forschungssystems ausmachen, wie Abbildung 1 illustriert.

Rund 70 Prozent der Forschung passiert innerhalb der Industrie und das heißt in Deutschland technologische Forschung im Wesentlichen im Fahrzeugbau, der Elektroindustrie, der Chemieindustrie und dem Maschinenbau zur Stärkung der Wettbewerbsfähigkeit der forschenden Unternehmen.
Abbildung 1: Aufteilung der Forschungsausgaben im deutschen Forschungssystem im Jahr 2007

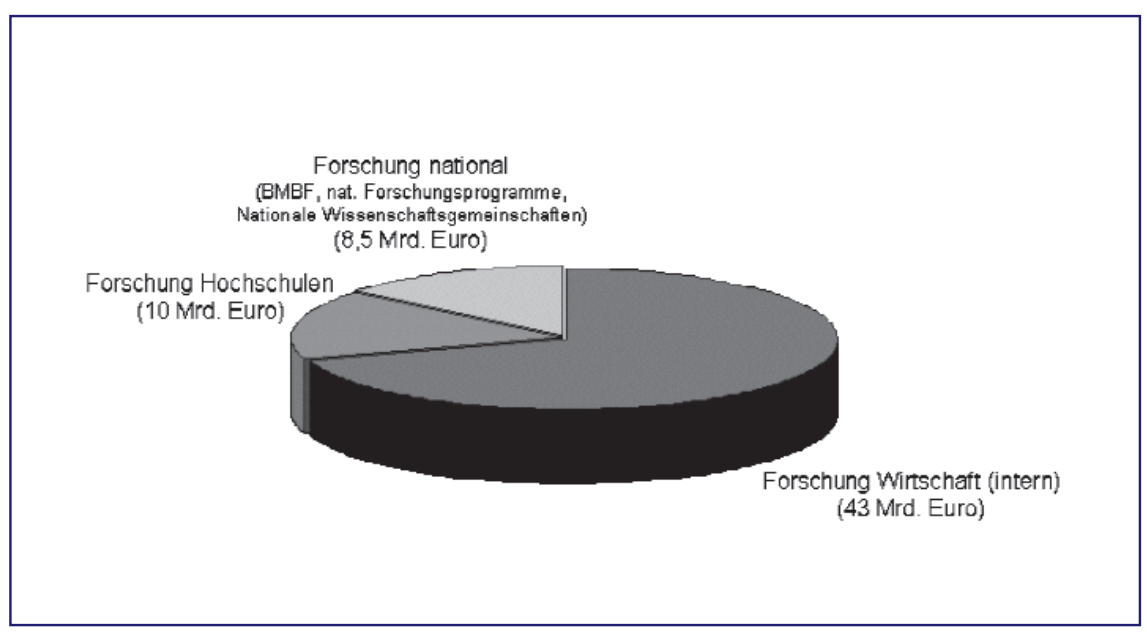

Quelle: Schneidewind (2009), auf Grundlage der Wissenschaftsstatistik (2008). 


\section{Hoffnungsschimmer für die zukünftige Entwicklung}

Es ist nicht gut bestellt um eine „Nachhaltige Wissenschaft“ in Deutschland. Dennoch sind Hoffnungsschimmer am Horizont zu erkennen, die es in den kommenden Jahren zu verstärken gilt:

- Wissenschaftsrat und DFG haben die Probleme einer zu disziplinären und zu wenig gesellschaftlich relevanten Ausrichtung des Forschungssystems erkannt. In der nächsten Runde der Exzellenzinitiative soll daher, wie jetzt schon im Rahmen einiger neuer DFG-Förderinstrumente, mehr Raum für transdisziplinäre Forschung und Forschungsverbünde geschaffen werden.

- Die nationalen Wissenschaftsgemeinschaften verstärken ihre Anstrengungen zu integrierten, interdisziplinären Programmen im Nachhaltigkeitsbereich. So existieren mehrere institutsübergreifende Programme in der Helmholtz-Gemeinschaft und auch die Fraunhofer-Gemeinschaft verfügt seit 2007 über eine Arbeitsgruppe und Initiative zur Bündelung der Nachhaltigkeitsforschung.

I Im außeruniversitären Bereich sind es aber immer noch die Institute außerhalb der nationalen Wissenschaftsgemeinschaften, die eine „Sustainability Science“ treiben: Ohne Institute wie das Institut für sozial-ökologische Forschung (ISOE) in Frankfurt, das Öko-Institut, das Institut für ökologische Wirtschaftsforschung (IÖW), das Wuppertal Institut und viele andere läge die Nachhaltigkeitswissenschaft in Deutschland noch weiter zurück. Daher gilt es den langfristigen Bestand dieser Institutslandschaft institutionell abzusichern.

- Im universitären Bereich sind es gerade einige der jungen und mittelgroßen Universitäten, insbesondere die Universitäten Kassel, Lüneburg und Oldenburg, die mit ihren ge- samtuniversitären Umwelt- und Nachhaltigkeitsstrategien, entsprechende Entwicklungen vorantreiben, die hoffentlich in Zukunft auf die eine oder andere Eliteuniversität überspringen werden.

\section{Anmerkung}

Der vorliegende Beitrag gibt komprimiert wichtige Ergebnisse zum Forschungssystem aus der Studie Schneidewind (2009) wieder. Die Studie selbst umfasst neben der Analyse des Forschungssystems auch eine Untersuchung „nachhaltiger Lehre“ im Bologna-System und entwickelt insbesondere Gestaltungsempfehlungen für einen nachhaltigkeitsorientierten Umbau des deutschen Wissenschaftssystems.

\section{Literatur}

Schneidewind, U.: Nachhaltige Wissenschaft. Plädoyer für einen Klimawandel im deutschen Wissenschafts- und Hochschulsystem. Marburg 2009.

Steinfeld, J. I. / Mino, T.: Education for sustainable development: the challenge of trans-disciplinarity. In: Sustainability Science 4/2009, S. 1-2.

Wissenschaftsstatistik: facts - Forschung \& Entwicklung: Zahlen und Fakten aus der Wissenschaftsstatistik $\mathrm{GmbH}$ im Stifterverband. 2008. Im Internet unter: http://www.stifterverband.de/-statistik_und_analysen/-publikationen/fue_facts/-fue_facts_12_2008.pdf.

\section{AUTOR + KONTAKT}

Dr. Uwe Schneidewind ist Professor für Betriebswirtschaftslehre an der Carl von Ossietzky-Universität Oldenburg. Von 2004 bis 2008 war er Präsident der Universität.

Universität Oldenburg, Department für Wirtschafts- und Rechtswissenschaften, Postfach 2503, 26111 Oldenburg, E-Mail: uwe.schneidewind@uni-oldenburg.de

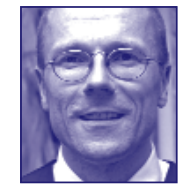

\section{Nachhaltigkeit}

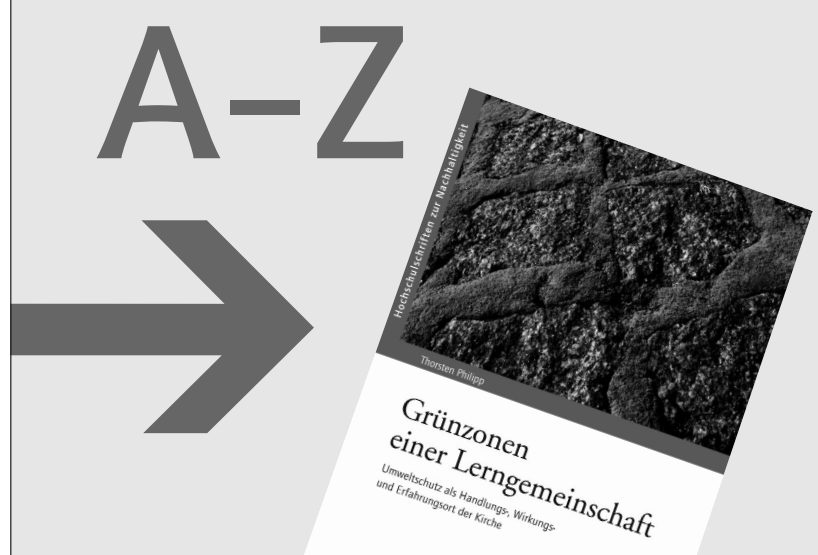

\section{S wie Schöpfungsverantwortung}

Die "ökologische Frage» verlangt nicht nur der Politik, sondern allen zivilgesellschaftlichen Akteur(inn)en neue Überlegungen ab. Gerade die katholische Kirche steht hier als weltweit größte Religionsgemeinschaft in einer besonderen Verantwortung. Der Politikwissenschaftler und Philologe Thorsten Philipp zeigt auf, welchen Stellenwert ökologische Fragen im Gefüge christlicher Sozialverkündigung einnehmen und welche Antworten die katholische Kirche liefert.

T. Phillipp

Grünzonen einer Lerngemeinschaft

Umweltschutz als Handlungs-, Wirkungs- und Erfahrungsort der Kirche Hochschulschriften zur Nachhaltigkeit Band 48

oekom verlag, München 2009, 233 Seiten, 39,90 EUR, ISBN 978-3-86581-177-6

Erhältlich bei

www.oekom.de

kontakt@oekom.de

Fax +49/(0)89/54 $4184-49$

Die guten Seiten der Zukunft

Ill oekom 
(c) 20I0 Authors; licensee IÖW and oekom verlag. This is an article distributed under the terms of the Creative Commons Attribution Non-Commercial No Derivates License (http://creativecommons.org/licenses/by-nc-nd/3.o/), which permits unrestricted use, distribution, and reproduction in any medium, provided the original work is properly cited. 